\title{
Improving Detection of Suicidal Ideation Among Depressed Patients in Primary Care
}

\author{
Paul A. Nutting, MD, MSPH ${ }^{1,2}$ \\ L. Miriam Dickinson, $P b D^{2}$ \\ Lisa V. Rubenstein, MD, MSPH \\ Robert D. Keeley, MD, MSPH \\ Jeffrey L. Smith, $P b D^{4}$ \\ Carl E. Elliott, $M S^{5}$ \\ ${ }^{1}$ Center for Research Strategies, Denver, \\ Colo \\ ${ }^{2}$ Department of Family Medicine, \\ University of Colorado Health Sciences \\ Center, Denver, Colo
}

${ }^{3}$ VA Greater Los Angeles Healthcare System and University of California, Los Angeles, Calif, and RAND Health Program, Santa Monica, Calif

${ }^{4}$ Mental Health QUERI, Central Arkansas Veterans Healthcare System, North Little Rock, Ark

${ }^{5}$ Colorado Foundation for Medical Care, Englewood, Colo

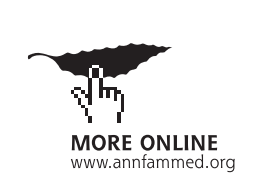

Conflicts of interest: none reported

\section{CORRESPONDING AUTHOR}

Paul A. Nutting, MD, MSPH

Center for Research Strategies

225 E 6th Ave, Suite 1150

Denver, CO 80203

Paul.Nutting@CRSLLC.org

\begin{abstract}
PURPOSE Primary care clinicians have difficulty detecting suicidal patients. This report evaluates the effect of 2 primary care interventions on the detection and subsequent referral or treatment of patients with depression and recent suicidal ideation.
\end{abstract}

METHODS Adult patients in 12 mixed-payer primary care practices and 9 not-forprofit staff model health maintenance organization (HMO) practices were screened for depression. Matched practices were randomized within plan type to intervention or usual care. The intervention for mixed-payer practices entailed brief training of physicians and office nurses to provide care management. The intervention for $\mathrm{HMO}$ practices consisted of guided development of quality improvement teams for depression care. A total of 880 enrolled patients met study criteria for depression, 232 of whom met criteria for recent suicidal ideation. Intervention effects on suicide detection and referral to mental health specialty care were evaluated with mixed-effects multilevel models in intent-to-treat analyses.

RESULTS Depressed patients with recent suicidal ideation were detected on $40.7 \%$ of index visits in intervention practices, compared with $20.5 \%$ in usual care practices (odds ratio $=2.64,95 \%$ confidence interval, 1.45-5.07), with HMO plan type and male sex associated with detection. The interventions had no effect on referral of patients, starting an antidepressant, or suicidal ideation reported at a 6-month follow-up, although power was limited for all 3 analyses.

CONCLUSIONS Primary care interventions to improve depression care can improve detection of recent suicidal ideation. Further work is needed to improve physician response to detection, including referral to specialty care and more aggressive treatment, and to observe the effect on outcomes.

Ann Fam Med 2005;3:529-536. DOI: 10.1370/afm.371.

\section{INTRODUCTION}

S uicide represents a major social ${ }^{1}$ and economic ${ }^{2,3}$ burden on the health of the American people and ranks among the top 10 causes of death for Americans aged 10 years and older. ${ }^{4}$ Suicide attempts are even more common, ${ }^{5 \cdot 7}$ increase morbidity and health care costs, ${ }^{3}$ and further elevate the risk of individuals for a subsequent completed suicide. ${ }^{8}$ Suicide prevention has been identified as a national priority ${ }^{9,10}$ and is now the target of a comprehensive national strategy. ${ }^{4}$

Primary care physicians have an important role in detecting patients at higher risk for suicide, and for prevention. Many ${ }^{11-14}$ but not all ${ }^{15}$ studies report that individuals make primary care visits before completing suicide. Improving primary care for suicidal patients poses several critical challenges. Completed suicide is relatively infrequent in primary care practice. Although suicidal ideation is more frequent than suicidal behavior or completed suicide, $^{16-21}$ the relationships among ideation, suicide attempts, and completed suicide are uncertain. ${ }^{5,6,8,22-27}$ Patients rarely volunteer suicidal ideation, although many will acknowledge ideation if asked directly ${ }^{13}$ and may want their physician to inquire. ${ }^{19}$ Although most primary care physicians believe in the value of detecting suicidal ideation, ${ }^{28,29}$ recent reports suggest that 
detection is accomplished inconsistently in practice. ${ }^{29-33}$ Rather than increasing primary care physician vigilance to suicide potential in all patients, ${ }^{31,34-39}$ a more targeted strategy would direct attention to patients with mental health problems among whom a large proportion of suicides occur. ${ }^{40-42}$ Major depression, for example, accounts for $60 \%$ of suicides ${ }^{42,44}$ and carries as much as a 20 -fold increase in lifetime risk of suicide. ${ }^{42-44}$

Recent research has demonstrated that a range of primary care interventions for depression reduce depression severity and increase functioning. ${ }^{45-51}$ This study compared the effect of different interventions for improving detection of suicidal ideation and referral to mental health specialty care among depressed patients with the effect of usual care.

\section{METHODS}

The Quality Enhancement by Strategic Teaming (QuEST) project and Mental Health Awareness Project (MHAP) were part of a collaboration of 4 depression trials, supported and coordinated by the National Institute of Mental Health, and were designed to have consistent measures and data collection procedures to allow planned meta-analyses such as that reported here. The methods used in QuEST ${ }^{45,51-54}$ and MHAP ${ }^{53,55}$ have been described previously and are summarized here. Both projects used a 4-level nested design, recruiting community-based health care programs, primary care practices within program, primary care clinicians within practice, and patients with depression within clinician. Participating practices were randomized to intervention or usual care conditions by a blocked randomization design that stratified practices into homogenous blocks and randomized within block. ${ }^{53}$ Except where noted, all measures and data collection procedures were identical in both projects.

The 2 improvement strategies, however, differed substantially. The QuEST intervention ${ }^{45,52,53}$ was carefully prescribed and consisted of training a practice nurse to deliver care management services by protocol. In contrast, the MHAP intervention ${ }^{53,55}$ focused on improving quality and trained practices to use 2 distinct team-based quality improvement approaches to formulate and implement specific improvement goals for depression care. Although both interventions included information about suicide in study materials, neither intervention provided additional specific training or support in suicide detection or prevention.

\section{Practices and Clinicians}

The QuEST study was conducted in 12 mixed-payer, community-based primary care practices. Each participating practice employed (1) 2 primary care physicians (family physicians and internists) willing to partici- pate in the study, (2) an office nurse willing to deliver the nursing intervention as detailed in the protocol if the practice was randomized to the enhanced care condition, (3) practice coordinators (administrative staff) willing to screen primary care patients for major depression as a part of routine care, and (4) no on-site mental health professional providing psychotherapy. Twenty-four primary care physicians (23 family physicians and 1 general internist) participated in the study.

The MHAP study was conducted in 9 primary care practices within 2 not-for-profit staff model health maintenance organizations (HMOs), 1 government funded (Veterans Affairs; 3 practices) and the other community based (Kaiser Permanente Medical Care Program of Northern California; 6 practices). Primary care practices within each organization were eligible to participate in the study if they were not currently participating in any depression quality improvement projects and if they could be matched on size, academic affiliation, patient population, and urban vs suburban location with at least 2 other practices. A total of 179 clinicians (family physicians, general internists, and nurse-practitioners) participated in the study.

\section{Patients}

Consecutive patients aged 18 years and older who were visiting each practice for a nonemergent condition were eligible for the study. A self-administered screening process identified individuals who reported that they felt sad, empty, or depressed, or had lost interest in things they normally enjoyed for 2 weeks or more during the past year and 1 week or more during the past month. Patients were subsequently confirmed to meet criteria for major depression with second-stage screening. Patients were excluded from the study if they had cognitive impairment that prevented them from completing the screener; had no access to a telephone or did not speak English with sufficient fluency to complete telephone interviews; indicated that they did not intend to receive care in the practice on an ongoing basis; were pregnant, were breastfeeding, or had given birth fewer than 3 months ago; or screened positive for current bereavement, lifetime mania, or lifetime alcohol dependence with current drinking.

Patients with suicidal ideation were not excluded from participation. ${ }^{53}$ Screening personnel in both usual care and intervention practices were given specific guidelines for notifying the physician when patients reported active suicidal ideation, but not passive thoughts of death. In both projects, physicians were equally likely to be notified in usual care and intervention conditions; however, physicians in the QuEST project were notified about suicidal ideation before the index visit, whereas physicians in the MHAP project were notified after the index visit. 
Study enrollment procedures, including management of suicidal intent, were approved by the Human Research Advisory Committee of the University of Arkansas for Medical Sciences and the Colorado Multi-institutional Review Board. The CONSORT (Consolidated Standards of Reporting Trials) flowcharts for patient recruitment and retention for both trials are shown as Supplemental Figures 1 and 2,

available online-only at http://www.annfammed. org/cgi/content/full/3/6/529/DC1.

\section{Training and Implementation of the Interventions}

In the QuEST project, all participating physicians and nurse care managers took part in 4 conference calls to become acquainted with the depression guidelines of the Agency for Health Care and Policy Research. ${ }^{35}$ The nurse care managers, who were all registered nurses, also completed an 8-hour training session on how to educate depressed patients about their treatment options, elicit treatment preferences, help patients overcome barriers to quality care, and monitor patients' response to treatment throughout the acute stage of care. The research team ensured nurse care managers met criteria to reliably administer the intervention at the conclusion of the training session and monitored their fidelity to the intervention after patient recruitment began. ${ }^{52,56}$ Consenting intervention patients were directly referred to the nurse care managers, who informed their physicians that the patient was depressed.

In the MHAP project, 9 practices were randomized within blocks to 2 types of quality improvement development teams or to usual care. ${ }^{55} \mathrm{MHAP}$ researchers provided each quality improvement team $41 / 2$ hours of interactive training on the evidence base for improving depression care. MHAP provided funds for quality improvement teams to meet for 16 hours to plan the quality improvement intervention programs. At the end of this period, each team submitted a written proposal for funding for additional planning and implementation support from their organizations. After approval, practices implemented the quality improvement interventions the teams had designed. Intervention teams did not systematically screen visiting patients for depression. MHAP evaluation data collectors enrolled consecutive visitors to intervention and usual care clinics, and did not refer patients in intervention clinics to those clinics' depression care interventions or otherwise notify the practices that the patient was depressed.

\section{Data Collection}

In both projects, enrolled patients underwent structured interviews at baseline and at 6 months by a trained interviewer blinded to the patient's experimental condi- tion. Participating clinicians completed a brief baseline survey describing their demographic characteristics and knowledge and beliefs about depression treatment. All patients consented to a review of their medical records to document selected elements of the process of care, including any mental health specialty referral made up to 6 months after the index visit.

\section{Operational Definition of Major Variables} in the Analysis

Recent suicidal ideation. Recent suicidal ideation for patients in both projects was defined as a positive response to any of the suicidal ideation questions on the modified Center for Epidemiologic Studies-Depression scale (mCES-D) $)^{57,58}$ at baseline. For the CES-D stem "I considered suicide," patients were included if they responded "some or a little of the time," "occasionally," or "most or all of the time" to the question "How often have you felt or behaved this way during the past week?" Suicidal ideation was assessed both at baseline and at the 6-month follow-up.

Severity of suicidal ideation. The World Health Organization Composite International Diagnostic Interview for Primary Care (CIDI) ${ }^{59}$ includes questions, "During the past 12 months, did you feel so low you thought a lot about committing suicide?" and "Did you make a plan as to how you might do it?" and "Did you attempt suicide?" Severity of suicidal ideation was measured on a 4-point scale based on the responses to the CIDI questions, with 4 being most severe and representing report of a suicide attempt.

Clinician detection of suicidal ideation. We defined clinician detection of suicidal ideation as a report by the patient at the baseline interview that the clinician asked during the index visit about thoughts of harming himself or herself.

Referral to a mental health specialist. Referral to mental health specialty care was determined by patient report at the baseline interview that a referral was made during the index visit, patient report at the 6-month interview that a referral had been made by 6 months after enrollment, or documentation of a referral or intent to refer in the medical record between baseline and 6 months after enrollment.

Treatment with antidepressants. Treatment with antidepressants was determined by patient report at the baseline and the 6-month interviews. A variable was created to characterize patients who started taking an antidepressant between baseline and 6 months.

Covariates. Social, demographic, and clinical covariates were collected from each patient at the baseline interview. Social and demographic covariates in the analyses included age, sex, minority status, insurance status (insured vs not), education (high school gradu- 
ate vs not), marital status (currently married vs not), annual household income, and a variable to control for differential patient lag in completing the baseline interview after the index visit. ${ }^{53}$ Clinical covariates included depression severity (based on the mCES-D), ${ }^{57,60}$ severity of suicidal ideation (based on response to the CIDI $)^{59}$ as described above, and physical comorbidity, measured by summing the total number of chronic physical conditions reported by the patient from among 14 conditions. Clinician covariates, collected at baseline, included age, sex, specialty, and clinician report of average time spent with a returning patient.

\section{Data Analysis}

We conducted intent-to-treat analyses in all patients with SAS 8.0 (SAS Institute, Cary, NC) using simple Mantel-Haenszel analysis and PROC MIXED or PROC GLIMMIX (to approximate the logistic regression model for dichotomous outcomes), comparing detection of recent suicidal ideation between intervention and usual care patients. Similar analyses compared referral to mental health specialty care and starting an antidepressant between patients with suicidal ideation who were and were not detected at the index visit. Finally, we constructed a similar model comparing suicidal thinking reported at 6 months between the patients who were and were not detected at baseline. We evaluated intervention effects on outcomes, controlling for the covariates, using mixed-effects multilevel (hierarchical) models in which patients were nested within clinicians, clinicians were nested within practice, and practices were nested within plan (mixed-payer practices in QuEST or $\mathrm{HMO}$ practices in MHAP) to account for any intraclass correlation at the clinician and practice levels.

Patient report of mental health specialty services in the prior 6 months was also used as a covariate in the detection analysis. The referral analysis excluded these patients. Univariate analysis, along with both forward and backward selection processes, was used to determine which covariates to retain in the final model for each outcome. The final model included intervention status (ie, usual care or intervention) along with all patient- and clinician-level social, demographic, and clinical covariates with $P$ values less than .20 in any prior analysis. Estimates of detection and referral rates for the usual care and intervention groups were obtained from the statistical models. Recruitment weights were used in all analyses to increase the representativeness of participating patients to eligible-to-participate patients. ${ }^{53}$

Power analyses indicated that our sample size was more than adequate for the detection analysis but provided limited power for the analysis of referral to mental health specialty care and starting an antidepres- sant. For the referral analysis, our data had $80 \%$ power to find significant differences only if the intervention doubled referral rates or if the rate of starting an antidepressant increased by an absolute $30 \%$ (eg, from $27 \%$ to $57 \%$ ). We also lacked power to detect an association between detection at baseline and suicidal thinking at 6 months, having only $80 \%$ power to observe a 21 -percentage point difference.

\section{RESULTS}

\section{Patient Characteristics}

The QuEST and MHAP projects enrolled 479 and 567 eligible patients, respectively (CONSORT Supplemental Figures 1 and 2). Of these, 939 (89.8\%) were followed up at 6 months (90.2\% in QuEST and $89.4 \%$ in MHAP), and of these patients, $93.7 \%$ (95.8\% in QuEST and 91.9\% in MHAP) had all the data items required for this analysis. Consequently, QuEST and MHAP contributed 414 and 466 patients, respectively, to the analysis.

Of the 880 patients, 232 (26.4\%) reported recent suicidal ideation (ie, occurring in the last 2 weeks) at the baseline interview (113 in QuEST and 119 in MHAP), and of these, $93(40.1 \%)$ reported a plan and $28(12.1 \%)$ reported an attempt during the previous 12 months. Fifty-eight percent reported receiving mental health specialty care in the prior 6 months. Among the patients with suicidal ideation, those in usual care and intervention practices were similar (Table 1), differing significantly only for marital status. Reflecting the different practice settings, patients differed more between projects; relative to MHAP patients, QuEST patients were significantly younger, more likely to be female, less educated with lower household incomes, and less likely to have health insurance.

\section{Detection of Suicidal Ideation}

Across both projects, patients with recent suicidal ideation were detected on $40.7 \%$ of index visits in intervention practices, compared with $20.5 \%$ in usual care practices (odds ratio $[\mathrm{OR}]=2.64,95 \%$ confidence interval, $1.45-5.07, P=.01)$. Differences in detection rates between the 2 projects-70.0\% vs $46.8 \%$ (QuEST) and $16.8 \%$ vs $7.1 \%$ (MHAP)—were probably due in part to the likelihood that QuEST clinicians were alerted at the index visit to those patients with active suicidal ideation (at most, 57 of the 113 patients with recent suicidal ideation in the QuEST project).

Male sex was the only patient characteristic associated with detection (Table 2). Depression severity, mental health specialty care in the prior 6 months, number of comorbid medical conditions, age, race, insurance status, and education did not predict detection, although severity of suicidal thinking showed a positive 


\begin{tabular}{|c|c|c|c|c|}
\hline \multirow[b]{2}{*}{ Characteristic } & \multicolumn{2}{|c|}{ By Trial Condition } & \multicolumn{2}{|c|}{ By Project } \\
\hline & $\begin{array}{l}\text { Intervention } \\
(\mathrm{n}=127)\end{array}$ & $\begin{array}{l}\text { Usual Care } \\
(n=105)\end{array}$ & $\begin{array}{l}\text { MHAP } \\
(n=119)\end{array}$ & $\begin{array}{l}\text { QuEST } \\
(n=113)\end{array}$ \\
\hline \multicolumn{5}{|l|}{ Social and demographic } \\
\hline Age, mean (SD), y & $45.9(12.1)$ & $45.9(11.5)$ & $49.6(11.4)^{*}$ & $42.0(11.0)^{*}$ \\
\hline Sex, \% female & 59.1 & 66.7 & $50.4^{*}$ & $75.2^{*}$ \\
\hline Minority status, \% minority & 20.5 & 23.8 & 26.1 & 17.7 \\
\hline Marital status, \% currently married & $41.4^{*}$ & $48.6 *$ & 40.3 & 44.3 \\
\hline High school educated, \% yes & 85.0 & 86.7 & $94.1^{*}$ & $77.0 *$ \\
\hline Employment, \% employed full or part time & 49.6 & 50.5 & 49.6 & 50.4 \\
\hline Health insurance, $\%$ with any & 88.6 & 90.5 & $100.0^{*}$ & $76.1^{*}$ \\
\hline Annual household income, mean (SD), \$ & $27,916(25,835)$ & $38,979(59,375)$ & $42,081 *(44,282)$ & $23,279 *(42,877)$ \\
\hline \multicolumn{5}{|l|}{ Clinical } \\
\hline $\begin{array}{l}\text { Medical comorbidity, mean (SD), No. of total } \\
\text { conditions reported }\end{array}$ & $2.36(1.82)$ & $2.58(1.88)$ & $2.64(2.05)$ & $2.27(1.60)$ \\
\hline $\begin{array}{l}\text { Severity of depression at baseline on } \mathrm{mCES}-\mathrm{D}^{\dagger}{ }^{\dagger} \text { mean } \\
\text { (SD) }\end{array}$ & $68.82(14.31)$ & $66.64(14.90)$ & $66.2(14.2)$ & $69.6(14.8)$ \\
\hline Mental health care in previous 6 months, \% yes & 58.3 & 57.1 & 60.5 & 54.9 \\
\hline Severity of suicidal ideation on CIDI, $\neq$ mean (SD) & $2.71(0.77)$ & $2.49(0.73)$ & $2.59(0.72)$ & $2.60(0.81)$ \\
\hline \multicolumn{5}{|c|}{$\begin{array}{l}\text { MHAP = Mental Health Awareness Project; QuEST = Quality Enhancement by Strategic Teaming; mCES-D = modified Center for Epidemiologic Studies-Depression scale; } \\
\text { CIDI = World Health Organization Composite International Diagnostic Interview for Primary Care. }\end{array}$} \\
\hline \multicolumn{5}{|l|}{$\begin{array}{l}\text { * Intervention and usual care differ at } P<.05 \text {. } \\
\dagger \text { Measured on a scale of } 0-100, \text { where } 100 \text { is most severe. } \\
\text { F Measured on a scale of } 1-4 \text {, where } 4 \text { is most severe (reportee }\end{array}$} \\
\hline
\end{tabular}

trend. Table 2 also confirms the effect of the intervention $(\mathrm{OR}=2.54)$ and the differential effect in the QuEST project sites $(\mathrm{OR}=3.12)$. Despite the different detection rates by project, the nonsignificant interaction term for intervention* project $(P=.99)$ suggests that the intervention had a significant effect in both projects. Models using MHAP data only, QuEST data only, and QuEST data excluding those patients for whom the physician might have been notified at the index visit yielded essentially similar results.

\section{Referral to Mental Health Specialty Care}

Among 98 patients with recent suicidal ideation and no mental health specialty care in the prior 6 months, $40.0 \%$ in usual care practices and $37.7 \%$ in intervention practices were referred $(P>.80)$. Neither plan type (mixed payer vs $\mathrm{HMO}$ ) or project (QuEST vs MHAP) $(P>.15$ for each $)$ nor depression severity $(P>.50)$ predicted referral. There was however a trend toward higher referral rates among more severely suicidal patients $(P<.10)$.

\section{Initiation of Antidepressant Therapy}

Among 84 patients with recent suicidal ideation and no antidepressant medication reported in the prior 6 months, $27 \%$ in usual care practices and $55 \%$ in intervention practices $(P=.12)$ started taking an antidepressant in the 6 months after detection.

\section{Suicidal Ideation at Follow-up}

Among the 199 patients with recent suicidal ideation at baseline who were in the study at the 6 -month followup, $40.0 \%$ who were detected and $35.6 \%$ who were not detected reported recent suicidal ideation at 6 months $(P=.52)$.

\section{DISCUSSION}

Two different approaches to improving primary care depression treatment significantly increased, and appeared to be comparably effective for, detection of depressed patients with recent suicidal ideation. This finding is encouraging and suggests an added value to interventions that are effective in improving depression care. Although just missing statistical significance, our results also suggest that clinicians are more likely to ask about suicidal thinking as the severity of depression increases, but not as the risk of suicidal ideation increases from passive thoughts of death to having a plan for suicide. The rates of detection were generally higher in the QuEST project than the MHAP project, which we attribute to the systematic notification of the physician about the patient's depression on the index visit in the former project.

Despite the lack of evidence that neither detection of suicidal ideation nor referral for specialty care is associated with improved outcomes, current guidelines 


\begin{tabular}{|c|c|c|}
\hline Characteristic* & $\begin{array}{l}\text { Adjusted OR } \\
(95 \% \mathrm{Cl})\end{array}$ & $P$ Value \\
\hline \multicolumn{3}{|l|}{ Patient: social and demographic } \\
\hline Male sex & $4.68(2.24-9.75)$ & $<.001$ \\
\hline \multicolumn{3}{|l|}{ Patient: clinical } \\
\hline Severity of depression on mCES-D & $1.01(0.98-1.03)$ & 6909 \\
\hline Severity of suicidal ideation on CIDI & $1.55(1.00-2.40)$ & .0506 \\
\hline Mental health specialty care in previous 6 months & $1.43(0.73-2.80)$ & .2994 \\
\hline $\begin{array}{l}\text { Project (QuEST vs MHAP), also plan type } \\
\text { (HMO vs mixed payer) }\end{array}$ & $3.12(2.12-4.59)$ & $<.001$ \\
\hline \multicolumn{3}{|l|}{ Intervention } \\
\hline Intervention vs usual care & $2.54(1.31-4.93)$ & .0059 \\
\hline Intervention* project interaction ${ }^{\dagger}$ & 1.01 & .9920 \\
\hline \multicolumn{3}{|c|}{$\begin{array}{l}\text { OR = odds ratio; Cl = confidence interval; mCES-D = modified Center for Epidemiologic Studies-Depres- } \\
\text { sion scale; } \mathrm{CIDI}=\text { World Health Organization Composite International Diagnostic Interview for Primary } \\
\text { Care; QuEST = Quality Enhancement by Strategic Teaming; MHAP = Mental Health Awareness Project; } \\
\text { HMO = health maintenance organization. }\end{array}$} \\
\hline \multicolumn{3}{|c|}{$\begin{array}{l}\text { * In prior analyses, none of the clinician characteristics met the level of significance required for inclusion } \\
\text { in this model }(P<.20) \text {. }\end{array}$} \\
\hline
\end{tabular}

of the interventions were responsible for the outcomes observed. Detection of recent suicidal ideation was, however, determined by patient report of physician behavior at a single visit. Consequently, we could not assess the clinician's suspicion of suicidality or suicidality assessments that occurred in other visits. Given that these patients were at elevated risk of harming themselves, however, clinician queries about suicidality at each visit may be necessary to ensure high-quality care. It would have been interesting to compare the relative effectiveness of the 2 interventions, but any conclusions would be potentially confounded by differences in the patient populations, health care systems, and reimbursement plans in which the interventions were tested. For the analyses of referral and starting an antidepressant among detected patients, the sample

for major depression recommend specialty referral for suicidal patients, particularly those with a suicide plan or suicide risk factors. ${ }^{35,36}$ It is disappointing that the intervention did not increase referral. Although the study had limited power, the absolute differences observed were modest, and severity of suicidal ideation was not associated with referral even in univariate analysis. Seeking evidence that primary care physicians may enhance care for these patients in other ways, we examined the association of detection on starting an antidepressant within the next 6 months and found a nonsignificant trend. The low response rates (specialty referral or starting an antidepressant) in the face of clinician sensitivity to suicidal ideation and low clinician discrimination of suicide risk severity requires further research. To reduce suicidal risk in depressed patients, either higher referral rates or better distinction among risk levels is needed. Patients most often do not mention suicide unless asked, and many completed suicides are not preceded by suicide attempts. We think that future depression interventions should focus specifically on primary care suicide prevention, including assessment of ideation and of level of risk, and an appropriate referral or therapeutic response.

The internal validity of our findings is strengthened by the use of a randomized block design to evaluate the intervention's ability to improve the process and outcomes of care using an intent-to-treat analysis that included patients who in fact did not receive ongoing intervention. We note that like many quality improvement investigations, our study had a design that did not allow us to draw conclusions about which component(s) size lacked power to fully investigate these effect of the intervention on responding to patients with suicidal ideation. Direct outcomes of suicidal detection were not readily available in our data as there were no reported suicides. Although we examined the effect of detection at baseline on subsequent suicidal ideation at the 6-month follow-up, our combined data set lacked power to detect a substantial effect. Consequently, these results should be considered preliminary until further research on the processes of depression care and their effect on response to suicidal ideation and outcomes is available.

External generalizability of our findings is enhanced because we found statistically comparable effects for 2 very different interventions tested in 21 practices operating within different organizational settings and serving socially and demographically diverse patients. Although we were not able to follow up all patients, our sample loss was smaller than that of most studies of this kind: $10.5 \%$ in MHAP and 9.3\% in QuEST at 6 months. ${ }^{54}$ We attempted to reduce the impact of sample loss by using attrition weights and modeling techniques that allowed us to project trends when patients did not complete the 6-month interview. ${ }^{54}$ External generalizability was also strengthened by the fact that interventions were implemented by the primary care practices themselves rather than by the research team, under naturalistic practice conditions whereby practices, clinicians, and patients were free to select the treatments they preferred. Although selection bias favoring practices and physicians with an interest in depression remains a possibility, there is evidence of similarity in 
practice and patient characteristics ${ }^{61,62}$ and physician clinical behavior ${ }^{63}$ between participants in primary care research networks and the larger universe of primary care. Exclusion of non-English-speaking patients may limit the generalizability of our results to some important subsets of the population.

In summary, our study demonstrates that 2 very different approaches to improving depression care in primary care settings can also improve clinician detection of suicidal ideation. These approaches can be further enhanced by adding components that add suicide detection and management to existing interventions, and observing the effect on outcomes.

To read or post commentaries in response to this article, see it online at http://www.annfammed.org/cgi/content/full/3/6/529.

Key words: Suicide; depression; primary care physicians; quality improvement

Submitted June 21, 2004; submitted, revised, April 24, 2005; accepted May 31, 2005.

Funding support: This research was supported by grant R01 MH54444 from the National Institute of Mental Health and by a grant from the John D. and Catherine T. MacArthur Foundation.

\section{References}

1. Palmer CS, Revicki DA, Halpern MT, Hatziandreu EJ. The cost of suicide and suicide attempts in the United States. Clin Neuropharmacol. 1995;18(Suppl 3):S25-S33.

2. Greenberg PE, Stiglin LE, Finkelstein SN, Berndt ER. The economic burden of depression in 1990. J Clin Psychiatry. 1993;54:405-418.

3. Rissmiller DJ, Steer R, Ranieri WF, Rissmiller F, Hogate P. Factors complicating cost containment in the treatment of suicidal patients. Hosp Community Psychiatry. 1994;45:782-788.

4. National Strategy for Suicide Prevention: Goals and Objectives for Action. Rockville, Md: US Department of Health and Human Services; 2001.

5. Weissman MM, Klerman GL, Markowitz JS, Ouellette R. Suicidal ideation and suicide attempts in panic disorder and attacks. N Engl J Med. 1989;321:1209-1214.

6. Vandivort DS, Locke BZ. Suicide ideation: its relation to depression, suicide and suicide attempt. Suicide Life Threat Behav. 1979;9:205-218.

7. Moscicki EK, O'Carroll P, Rae DS, et al. Suicide attempts in the Epidemiologic Catchment Area Study. Yale J Biol Med. 1988;61:259-268.

8. Leon AC, Friedman RA, Sweeney JA, Brown RP, Mann JJ. Statistical issues in the identification of risk factors for suicidal behavior: the application of survival analysis. Psychiatry Res. 1990;31:99-108.

9. Mental Health: A Report of the Surgeon General. Rockville, Md: US Department of Health and Human Services; 1999.

10. US Public Health Service. The Surgeon General's Call to Action to Prevent Suicide. Washington, DC: US Department of Health and Human Services; 1999.

11. Frierson RL. Suicide attempts by the old and the very old. Arch Intern Med. 1991;151:141-144.

12. Meats $P$, Solomka BA. A perspective on suicides in the ' 90 s. Psychiatr Bull. 1995; 19:666-669.

13. Vassilas CA, Morgan HG. General practitioners' contact with victims of suicide. BMJ. 1993;307:300-301.
14. Appleby L, Amos T, Doyle U, Tomenson B, Woodman M. General practitioners and young suicides: a preventive role for primary care. Br J Psychiatry. 1996;168:330-333.

15. Lin $\mathrm{EH}$, Von Korff $M$, Wagner $E H$. Identifying suicide potential in primary care. J Gen Intern Med. 1989;4:1-6.

16. Olfson M, Weissman MM, Leon AC, Sheehan DV, Farber L. Suicidal ideation in primary care. J Gen Intern Med. 1996;11:447-453.

17. Lish JD, Zimmerman M, Farber NJ, et al. Suicide screening in a primary care setting at a Veterans Affairs Medical Center. Psychosomatics. 1996;37:413-424.

18. Callahan CM, Hendrie HC, Nienaber NA, Tierney WM. Suicidal ideation among older primary care patients. J Am Geriatr Soc. 1996;44:1205-1209.

19. Zimmerman M, Lish JD, Lush DT, et al. Suicidal ideation among urban medical outpatients. J Gen Intern Med. 1995;10:573-576.

20. Cappelli M, Clulow MK, Goodman JT, et al. Identifying depressed and suicidal adolescents in a teen health clinic. J Adolesc Health. 1995; $16: 64-70$

21. Cooper-Patrick L, Crum RM, Ford DE. Identifying suicidal ideation in general medical patients. JAMA. 1994;272:1757-1762.

22. Garland AF, Zigler E. Adolescent suicide prevention: current research and social policy implications. Am Psychol. 1993;48:169-182.

23. Friedman JM, Asnis GM, Boeck M, DiFiore J. Prevalence of specific suicidal behaviors in a high school sample. Am J Psychiatry. 1987:144:1203-1206.

24. Beck AT, Steer RA, Kovacs M, Garrison B. Hopelessness and eventual suicide: a 10-year prospective study of patients hospitalized with suicidal ideation. Am J Psychiatry. 1985;142:559-563.

25. Fergusson DM, Lynskey MT. Suicide attempts and suicidal ideation in a birth cohort of 16-year-old New Zealanders. J Am Acad Child Adolesc Psychiatry. 1995;34:1308-1317.

26. Kosky R, Silburn S, Zubrick SR. Are children and adolescents who have suicidal thoughts different from those who attempt suicide? J Nerv Ment Dis. 1990;178:38-43.

27. Goldstein RB, Black DW, Nasrallah A, Winokur G. The prediction of suicide: sensitivity, specificity, and predictive value of a multivariate model applied to suicide among 1906 patients with affective disorders. Arch Gen Psychiatry. 1991;48:418-422.

28. Williams JW Jr, Rost K, Dietrich AJ, et al. Primary care physicians' approach to depressive disorders: effects of physician specialty and practice structure. Arch Fam Med. 1999;8:58-67.

29. Frankenfield DL, Keyl PM, Gielen A, et al. Adolescent patientshealthy or hurting? Missed opportunities to screen for suicide risk in the primary care setting. Arch Pediatr Adolesc Med. 2000;154:162-168

30. Uncapher $H$, Arean PA. Physicians are less willing to treat suicidal ideation in older patients. J Am Geriatr Soc. 2000;48:188-192.

31. Milton J, Ferguson B, Mills T. Risk assessment and suicide prevention in primary care. Crisis. 1999;20:171-177.

32. Stoppe G, Sandholzer H, Huppertz C, Duwe H, Staedt J. Family physicians and the risk of suicide in the depressed elderly. J Affect Disord. 1999;54:193-198.

33. Kaplan MS, Adamek ME, Calderon A. Managing depressed and suicidal geriatric patients: differences among primary care physicians. Gerontologist. 1999;39:417-425.

34. Summary of the practice parameters for the assessment and treatment of children and adolescents with suicidal behavior. J Am Acad Child Adolesc Psychiatry. 2001;40:495-499.

35. Depression Guideline Panel. Depression in Primary Care: Volume 1. Detection and Diagnosis. Clinical Practice Guideline Number 5. Rockville, Md: US Department of Health and Human Services, Public Health Service, Agency for Health Care Policy and Research; 1993. AHCPR publication No. 93-0550. 
36. Depression Guideline Panel. Depression in Primary Care: Volume 2. Treatment of Major Depression. Clinical Practice Guideline Number 5. Rockville, Md: US Department of Health and Human Services, Public Health Service, Agency for Health Care Policy and Research; 1993. AHCPR Publication No. 93-0551.

37. Wilkinson G. Can suicide be prevented? Better treatment of mental illness is more appropriate aim. BMJ. 1994;309:860-861.

38. Geddes J. Suicide and homicide by people with mental illness. BMJ. 1999;318:1225-1226.

39. Matthews K, Milne S, Ashcroft GW. Role of doctors in the prevention of suicide: the final consultation. Br J Gen Pract. 1994;44:345-348.

40. Carlson GA, Rich CL, Grayson P, Fowler RC. Secular trends in psychiatric diagnoses of suicide victims. J Affect Disord. 1991;21:127-132.

41. Harris EC, Barraclough B. Suicide as an outcome for mental disorders: a meta-analysis. Br J Psychiatry. 1997;170:205-228.

42. Gaynes BN, West SL, Ford CA, et al. Screening for suicide risk in adults: a summary of the evidence for the U.S. Preventive Services Task Force. Ann Intern Med. 2004;140:822-835.

43. Stoudemire A, Frank R, Hedemark N, Kamlet M, Blazer D. The economic burden of depression. Gen Hosp Psychiatry. 1986;8:387-394.

44. Angst J, Angst F, Stassen HH. Suicide risk in patients with major depressive disorder. J Clin Psychiatry. 1999;60(Suppl 2):57-62.

45. Rost K, Nutting P, Smith J, Werner J, Duan N. Improving depression outcomes in community primary care practice: a randomized trial of the QuEST intervention. Quality Enhancement by Strategic Teaming. J Gen Intern Med. 2001;16:143-149.

46. Shye D, Brown JB, Mullooly JP, Nichols GA. Understanding changes in primary care clinicians' satisfaction from depression care activities during adoption of selective serotonin reuptake inhibitors. Am J Manag Care. 2002;8:963-974.

47. Katzelnick DJ, Simon GE, Pearson SD, et al. Randomized trial of a depression management program in high utilizers of medical care. Arch Fam Med. 2000;9:345-351.

48. Simon GE, VonKorff M, Rutter C, Wagner E. Randomised trial of monitoring, feedback, and management of care by telephone to improve treatment of depression in primary care. BMJ. 2000:320:550-554

49. Wells KB, Sherbourne C, Schoenbaum M, et al. Impact of disseminating quality improvement programs for depression in managed primary care: a randomized controlled trial. JAMA. 2000;283:212-220.
50. Schulberg HC, Block MR, Madonia MJ, et al. Treating major depression in primary care practice: eight-month clinical outcomes. Arch Gen Psychiatry. 1996;53:913-919.

51. Hunkeler EM, Meresman JF, Hargreaves WA, et al. Efficacy of nurse telehealth care and peer support in augmenting treatment of depression in primary care. Arch Fam Med. 2000;9:700-708.

52. Rost K, Nutting PA, Smith J, Werner JJ. Designing and implementing a primary care intervention trial to improve the quality and outcome of care for major depression. Gen Hosp Psychiatry. 2000;22:66-77.

53. Rost KM, Duan N, Rubenstein LV, et al. The Quality Improvement for Depression collaboration: general analytic strategies for a coordinated study of quality improvement in depression care. Gen Hosp Psychiatry. 2001;23:239-253.

54. Rost K, Nutting P, Smith JL, Elliott CE, Dickinson M. Managing depression as a chronic disease: a randomised trial of ongoing treatment in primary care. BMJ. 2002;325:934.

55. Rubenstein LV, Parker LE, Meredith LS, et al. Understanding teambased quality improvement for depression in primary care. Health Serv Res. 2002;37:1009-1029.

56. Smith JL, Rost KM, Nutting PA, Elliott CE, Duan N. A primary care intervention for depression. J Rural Health. 2000;16:313-323.

57. Radloff LS. The CES-D scale: a self-report depression scale for research in the general population. Appl Psychol Meas. 1977;1:385-401.

58. Myers JK, Weissman MM. Use of a self-report symptom scale to detect depression in a community sample. Am J Psychiatry. 1980;137:1081-1084.

59. Composite International Diagnostic Interview for Primary Care, Version 2.0. Geneva, Switzerland: World Health Organization; 1996.

60. Orlando M, Sherbourne CD, Thissen D. Summed-score linking using item response theory: application to depression measurement. Psychol Assess. 2000;12:354-359.

61. Green LA, Miller RS, Reed FM, Iverson DC, Barley GE. How representative of typical practice are practice-based research networks? A report from the Ambulatory Sentinel Practice Network Inc (ASPN). Arch Fam Med. 1993;2:939-949.

62. Gilchrist V, Miller RS, Gillanders WR, et al. Does family practice at residency teaching sites reflect community practice? J Fam Pract. 1993;37:555-563.

63. Nutting PA, Baier M, Werner JJ, et al. Practice patterns of family physicians in practice-based research networks: a report from ASPN. Ambulatory Sentinel Practice Network. J Am Board Fam Pract. 1999:12:278-284. 